\title{
Meaningful Integration of Data Analytics and Services in MIDAS Project: Engaging Users in the Co-Design of a Health Analytics Platform
}

\author{
Brian Cleland \\ Ulster University \\ Jordanstown Campus \\ Northern Ireland \\ b.cleland@ulster.ac.uk
}

Michaela Black

Ulster University

Magee Campus

Northern Ireland

mm.black@ulster.ac.uk

Julius Francis Gomes

University of Oulu

Oulu

Finland

Julius.FrancisGomes@oulu.fi

\author{
Jonathan Wallace \\ Ulster University \\ Jordanstown Campus \\ Northern Ireland \\ jg.wallace@ulster.ac.uk
}

\author{
Maurice Mulvenna \\ Ulster University \\ Jordanstown Campus \\ Northern Ireland \\ md.mulvenna@ulster.ac.uk
}

\author{
Peter Connolly \\ Health Service Executive \\ Dublin \\ Ireland \\ Peter.Connolly@hse.ie
}

\author{
Raymond Bond \\ Ulster University \\ Jordanstown Campus \\ Northern Ireland \\ rb.bond@ulster.ac.uk
}

\author{
Deborah Rankin \\ Ulster University \\ Magee Campus \\ Northern Ireland \\ d.rankin1@ulster.ac.uk
}

\author{
Gorka Epelde \\ Vicomtech \\ San Sebastian \\ Basque Country, Spain \\ gepelde@vicomtech.org
}

\author{
Susan Campbell \\ Business Services Organisation \\ Belfast \\ Northern Ireland \\ Susan.Campbell@hscni.net
}

\begin{abstract}
This paper outlines the scope and aims of the MIDAS Project, a Horizon 2020-funded initiative to develop a data analytics platform to support better policy-making in the European health sector. It focuses specifically on the engagement of users in the co-design of the platform, and describes a participatory workshop which encouraged stakeholders to share their understanding of the problem to be addressed and insights into potential solutions. The major elements of the workshop are described and the key results are presented. Participant feedback is analysed and the main lessons and insights are highlighted, including the importance of an adopting an iterative approach to user engagement in software design and development.
\end{abstract}

Big data, data analytics, health, health policy, user-centred design, co-design, agile development

\section{INTRODUCTION}

The MIDAS Project (Meaningful Integration of Data Analytics and Services) is a 40-month Horizon 2020 -funded project to address the needs of policymakers and citizens through the delivery of a unified big data platform. In order to ensure that the information provided by the MIDAS platform is actionable, the technical implementation has adopted user-centred design approach to maximise the impact on health policy across Europe. The MIDAS consortium includes partners from six European countries, plus the U.S., with a wide range of experts from academia, business and the public. By including as broad a range of expertise as possible in the design of the system, the project aims to effectively meet user needs and to be as usable as possible. This is particularly challenging given the wide range of potential users of the system: although the primary beneficiaries of platform are policy-makers, potential users might include stakeholders with many different levels of technical knowledge and needs - from data scientists, to statisticians, to politicians. 
Brian Cleland • Jonathan Wallace $\bullet$ Raymond Bond $\bullet$ Michaela Black $\bullet$ Maurice Mulvenna $\bullet$ Deborah Rankin $\bullet$ Julius Francis Gomes • Peter Connolly • Gorka Epelde • Susan Campbell

A report by McKinsey (Kayyali et al., 2013) entitled "The Big Data Revolution in Health Care: Accelerating Value and Innovation" sets the context for this work: firstly, in terms of demand, there is significant economic pressure on existing healthcare budgets to derive more value from data, and to better inform policy-making with data that has already been warehoused but has not yet been used for operational decision-making (i.e., secondary purposes). Secondly, in terms of the supply of relevant data, there is an increasing flow of data from clinical sources and personal data banks (for example, social media and the quantified self). Thirdly, governments are catalysing market change with initiatives in open data. Finally, technical capabilities are rapidly improving regarding the secure storage and management of heterogeneous data. These circumstances have provided fertile ground for innovation. Specifically, The MIDAS proposal represents innovation in the utilisation of big healthcare-related data, since a tipping point is being brought about by the convergence of multiple positive changes in demand, supply, policy and technology as outlined above.

Although governments, technology providers and other stakeholder may take a different perspective on what big data is, it has been agreed that the MIDAS platform will include:

- Components that facilitate the collection and preparation of heterogeneous data;

- Architectures for data storage, data integration, data virtualization, data cleansing, deployment and management;

- 'Privacy by design' models to anonymise and aggregate data making it interoperable to the analytics tools and to provide multisite predictive modules.

- Tools and algorithms that extract meaning from data, including machine learning and other data analytics techniques;

- Components that either visualise or serves out the aggregated data and outputs from the analytics to management dashboards and intuitive interactive visualisation aids to provide actionable insights.

Given the heterogeneous nature of the various data sources, policy environments and stakeholder perspectives, MIDAS has adopted an agile, usercentred design approach in order to ensure that user needs are met across the consortium. Based on this participatory ethos, the MIDAS Project held a CoDesign Workshop on 16 November 2017, which was open to consortium members as well as the wider public. The workshop took participants through a staged process, which included the development of "personas" (i.e., typical users of the system), the identification of "user stories" (simple, non-technical descriptions of user requirements), and the brainstorming of "wireframes" (interface design ideas) on paper and online. In order to make the event as engaging as possible, participants were grouped into small, collaborative teams, with each team containing a mix of experience and expertise. The results from the workshop were subsequently collated, analysed and distributed among consortium partners to inform the future development of the MIDAS platform. In this paper we will outline the methodology adopted for the workshop, summarise the key results, and discuss the main insights and lessons learned.

\section{METHODOLOGY}

The workshop was attended by approximately 80 participants, including a mixture of consortium members and external stakeholders. The professional backgrounds of attendees were diverse and included policy-makers, civil servants, academic experts, and industry representatives. Participants were divided into nine tables, with each table comprising a mixture of different users and stakeholders. Each table also had an allocated facilitator, equipped with a laptop, who was charged with recording the discussion within that group. Facilitators, selected from the consortium members and given training prior to the event, were responsible for supporting discussion and engagement at each table, while also recording information generated by the group.

The overall structure of the workshop was intended to lead potential users of the MIDAS system through a logical series of stages, from understanding the characteristics of typical users (i.e., creating personas), to identifying scenarios in which the system might be deployed (i.e., user stories), to manually visualising interface components and layouts, and finally to converting these paper-based sketches into more detailed digital wireframes. This approach was based on industry-standard methods which have been widely adopted across the software sector. Since capturing user needs can be a challenging task (e.g., von Hippel, 1994; Bogers et al., 2010), a structured approach was adopted in order to generate as much value as possible in the limited time available. This also had the benefit of educating users about the nature of user-centred design techniques, creating an opportunity for them to bring useful knowledge back to their own organisations.

The key elements of the workshop structure are outlined below:

User Personas - defined as 'fictitious, specific, concrete representations of target users' by Pruitt \& Adlin (2006) - have been a common feature of industrial product design since their introduction by Cooper (1999), are widely regarded as a major component of user-centred design (Miaskiewicz and 
Brian Cleland $\bullet$ Jonathan Wallace $\bullet$ Raymond Bond $\bullet$ Michaela Black $\bullet$ Maurice Mulvenna $\bullet$ Deborah Rankin $\bullet$ Julius Francis Gomes • Peter Connolly • Gorka Epelde • Susan Campbell

Kozar, 2011). In the co-design workshop, the analysis of user personas was divided into two parts: firstly, each table evaluated five pre-existing key personas that had been developed by the MIDAS team; secondly each table was asked to come up with a novel user persona. In this way, it was hoped that assumptions about the user base would be challenged, and potential gaps would be identified and filled.
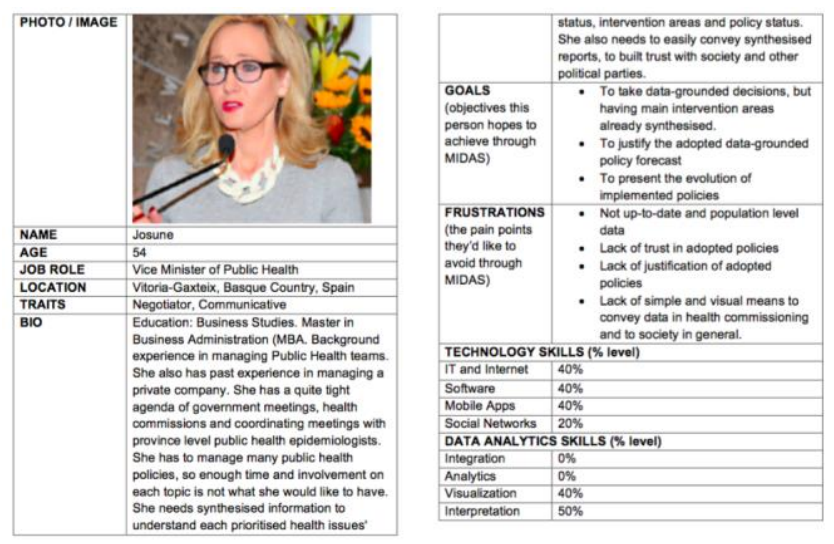

Figure 1: Example MIDAS user persona.

User Stories are brief narratives that describe a specific work activity of a single worker and situation. User stories generally conform to the following format: "As a <type of user>, I want <some goal> so that <some reason>" (Turner et al., 2013). For the user stories element of the workshop, each group was given a short period of time to develop user stories for each of the five preexisting personas plus the new persona that they had created. The aim of this task was to ensure that a variety of user stories were created for each of the major personas, as well as for any new personas that had been generated.

Wireframing is a simple visual description of the elements of a web page, or for an application screen (Puerta et al, 2005). For the purposes of the workshop, wireframing was split into two parts. In the first session, users were asked to select a user story from the previous activity, and then to collaborate on a paper-based sketch of what a relevant screen layout should look like. In the second part of the wireframing activity, each table facilitator was asked to take the paper wireframes and convert those into a digital equivalent using an open source software application. This stage of the co-design process was intended to produce semirealistic design concepts that could feed into the future development of the MIDAS platform.

Table 1: MIDAS Co-Design Workshop Agenda

\begin{tabular}{|c|c|}
\hline Time & \multicolumn{1}{c|}{ Session } \\
\hline 10:00 & Stage 1: User Personas \\
\hline
\end{tabular}

\begin{tabular}{|l|l|}
\hline 10:45 & Stage 2: User Stories \\
\hline 11:30 & Tea/Coffee, Technical Demos \\
\hline 11:45 & Stage 3: Paper-based wireframing \\
\hline 12:30 & Stage 4: Digital wireframing \\
\hline $13: 15$ & Lunch \\
\hline $14: 15$ & Final Presentations \\
\hline $15: 00$ & Close \\
\hline
\end{tabular}

\section{RESULTS}

Extensive feedback was received on the five user personas designed by the MIDAS team prior to the workshop. In general, participants agreed that these personas were representative of the wider user base. In addition, nine new personas were generated. For each of these pre-existing and new personas a range of user stories were created, with some tables providing multiple stories for each persona. 22 paper wireframes were created based on the user stories, and from those paper-based designs 16 digital wireframes were ultimately derived. Examples of paper and digital wireframes are shown in Figures 2 and 3.

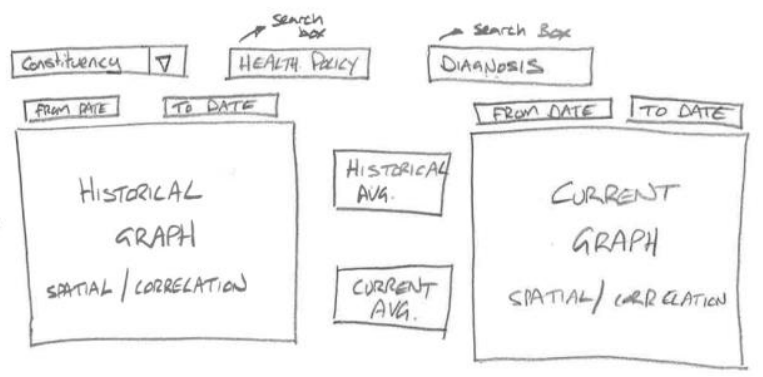

Figure 2: An example of a paper-based wireframe design from the workshop.


Figure 3: An example of a digitised wireframe design from the workshop. 
Brian Cleland $\bullet$ Jonathan Wallace $\bullet$ Raymond Bond $\bullet$ Michaela Black $\bullet$ Maurice Mulvenna $\bullet$ Deborah Rankin $\bullet$ Julius Francis Gomes • Peter Connolly • Gorka Epelde • Susan Campbell

At the end of the workshop, a survey was carried out to collect feedback from participants on their experience of the event. Attendees were asked to state which parts of the workshop worked well, which parts of the workshop could be improved, and whether they would consider using co-design methods in their own organisation. Responses to the survey were generally favourable, with most participants stating that they found the process both educational and enjoyable. Some noted that they derived more benefit from the experience than they had expected. Areas for improvement included the fact that some participants started to get tired towards the end. Some respondents commented that the presentations from each table at the end of the event were unnecessary. Others expressed the view that more information on the MIDAS platform, including more time available to interact with technical demos and prototypes, would have been helpful.

\section{DISCUSSION AND CONCLUSION}

One of the suggestions that came up in the participant survey was that the workshop might be repeated in other regions, or that the results might be validated by MIDAS user organisations. The need to iterate within the design process and to regularly re-evaluate findings is strongly supported by the literature on agile development methods. As Ferreira et al. (2007) point out, iterating on design processes can facilitate usability testing, support the incorporation of results into future development cycles and enhance the relationship between designers and developers. While the results of the workshop were shared and discussed with the MIDAS developer network, it is likely that further workshop sessions to evaluate ongoing design decisions would strengthen the user-centredness of the final system. Indeed, without such iterative evaluation processes it is possible that useful insights might be forgotten, or that sub-optimal design decisions might not be identified in a timely fashion.

Overall, the feedback from both MIDAS partners and non-MIDAS stakeholders was that the workshop was a valuable exercise. Many nontechnical participants appeared to find the codesign experience both enjoyable and educational. Future workshops should take into account the fact that the agenda could be improved, particularly with regard to the later wireframing and presentation sessions. While the digitisation of wireframes was helpful in moving from a "sketchy" illustration to a more concrete representation, there was a consensus that the final presentation of results by each table was not necessary or of particular interest to participants. Instead, more time could have been allocated to engaging technical stakeholders in a structured analysis of the results, with a view to ensuring that key insights were integrated into the future development of the system. This, together with an iterative approach to user engagement, would help to maximise the value to both users and developers of the MIDAS platform.

\section{ACKNOWLEDGEMENTS}

The MIDAS Consortium gratefully acknowledge the support to this project from the European Union research fund 'Big Data Supporting Public Health Policies', under Grant Agreement No. 727721 (H2020-SC1-2016-CNECT SC1-PM-18-2016).

\section{REFERENCES}

Bogers, M., Afuah, A., \& Bastian, B. (2010). Users as innovators: A review, critique, and future research directions. Journal of management, 36(4), 857-875.

Da Silva, T. S., Martin, A., Maurer, F., \& Silveira, M. (2011, August). User-centered design and agile methods: a systematic review. In Agile Conference (AGILE), 2011 (pp. 77-86). IEEE.

Ferreira, J., Noble, J., \& Biddle, R. (2007, August). Agile development iterations and UI design. In Agile Conference (AGILE), 2007 (pp. 50-58). IEEE.

Kayyali, B., Knott, D., \& Van Kuiken, S. (2013). The big-data revolution in US health care: Accelerating value and innovation. Mc Kinsey \& Company, 2(8), 1-13.

Miaskiewicz, T., \& Kozar, K. A. (2011). Personas and user-centered design: How can personas benefit product design processes?. Design Studies, 32(5), 417-430.

Pruitt, J., \& Grudin, J. (2003, June). Personas: practice and theory. In Proceedings of the 2003 conference on Designing for user experiences (pp. 1-15). ACM.

Puerta, A., Micheletti, M., \& Mak, A. (2005, January). The UI pilot: a model-based tool to guide early interface design. In Proceedings of the 10th international conference on Intelligent user interfaces (pp. 215-222). ACM.

Turner, A. M., Reeder, B., \& Ramey, J. (2013). Scenarios, personas and user stories: Usercentered evidence-based design representations of communicable disease investigations. Journal of biomedical informatics, 46(4), 575-584.

Von Hippel, E. (1994). "Sticky information" and the locus of problem solving: implications for innovation. Management science, 40(4), 429439. 